\title{
COMPARABILIDADE X UNIFORMIDADE DOS RELATÓRIOS FINANCEIROS: UMA ANÁLISE EMPÍRICA DURANTE O PERÍODO DE TRANSIÇÃO REGULATÓRIA DA CONTABILIDADE BRASILEIRA AO PADRÃO INTERNACIONAL
}

\author{
Alex Mussoi Ribeiro ${ }^{1}$ \\ Carlos Henrique Silva do Carmo ${ }^{2}$ \\ Luiz Paulo Lopes Fávero ${ }^{3}$ \\ Luiz Nelson Guedes de Carvalho ${ }^{4}$
}

\begin{abstract}
Resumo: O objetivo desta pesquisa foi testar se existem diferenças significativas entre o comportamento da comparabilidade e da uniformidade dos relatórios financeiros durante e após o período de adoção das normas internacionais de contabilidade no Brasil. As companhias analisadas foram as de capital aberto com dados disponíveis durante o período 2002 a 2012. A medida de comparabilidade utilizada foi a similaridade da função contábil de DeFranco, Kothari e Verdi (2011) e a de uniformidade foi a comovimentação dos lucros de Lang, Maffett e Owens (2010). Os resultados encontrados comprovaram para as empresas analisadas que a comparabilidade e a uniformidade possuem comportamentos distintos durante o período de alteração regulatória que ocorreu na contabilidade brasileira. Enquanto a comparabilidade obteve um ganho significativo após a adoção das normas internacionais, a uniformidade teve uma queda significativa durante a transição e após a adoção do padrão internacional no Brasil. Esta pesquisa demonstrou que a comparabilidade dos relatórios financeiros não pode ser confundida com a sua uniformidade e que o aumento da variabilidade das escolhas dos gestores tende a diminuir a uniformidade, mas não a comparabilidade dos relatórios financeiros.
\end{abstract}

Palavras-chave: Comparabilidade. Regulação. IFRS. Uniformidade.

E-mail: areksu@terra.com.br - Universidade Federal de Goiás

2 E-mail: chscarmo@ufg.br - Universidade Federal de Goiás

${ }^{3}$ E-mail: Ipfavero@usp.br - Universidade de São Paulo

${ }^{4}$ E-mail: Inelson@usp.br - Universidade de São Paulo 


\title{
COMPARABILITY VERSUS UNIFORMITY OF FINANCIAL REPORTING: AN EMPIRICAL ANALISYS OF BRAZILIAN COMPANIES TRANSITION TO
} IFRS

\begin{abstract}
The objective of this research was to verify the differences between the behavior of the comparability and the uniformity of financial reporting during and after the adoption of international accounting standards in Brazil. The universe analyzed was coumposed by publicly traded companies with available data for the period 2002 to 2012. The comparability of financial reporting was represented unsing the similarity of the accounting function model developed by DeFranco, Kothari and Verdi (2011) and the uniformity was represented with the earnings co-movement model of Lang, Maffett and Owens (2010). The results have shown that comparability and uniformity of financial reports have distinct behaviors during the trasition period and after the full adoption of international accounting standards in Brazil. While comparability obtained a significant gain after the full adoption of international standards, uniformity had a significant drop during the transition and after the adoption of the international accounting standard in Brazil. This research showed that the comparability of financial reports should not be confused with it's uniformity and that an increase in the variability of choices of managers tends to decrease uniformity but not the comparability of financial reports.
\end{abstract}

Keywords: Comparability. Regulation. IFRS. Uniformity. 


\section{INTRODUÇÃO}

0 cenário regulatório da contabilidade financeira passou por uma grande revolução ao redor do mundo com a adoção do padrão internacional de contabilidade emitido pelo International Accounting Standards Board (IASB). Diversas economias mundiais, incluído o Brasil, trocaram a sua soberania na emissão de normativos que regulam a forma como as companhias devem fazer a sua contabilidade em detrimento de uma maior harmonia mundial nas práticas de relatórios financeiros. O principal argumento defendido pelos envolvidos com a internacionalização dos padrões contábeis foi o ganho de comparabilidade dos relatórios financeiros e como isto iria facilitar o processo de alocação de capital entre fronteiras, trazendo maiores retornos aos investidores e uma possibilidade maior de diversificação de suas carteiras (Tweedie, 2007; Securities and Exchange Commission (SEC), 2010; Hail, Leuz \& Wysocki, 2010; Joos \& Leung, 2011).

Ao analisar a relevância da comparabilidade dentro do modelo decisório dos investidores, percebe-se que ela é o centro das atenções (SEC, 2000; Chartered Financial Analyst (CFA), 2007 ). O produto final da contabilidade é uma informação (por exemplo, o lucro) que só é útil quando inserida no modelo decisório de algum de seus usuários. Sobre isto, Scott (2009) afirma que informação é uma evidência com o potencial de afetar as decisões dos indivíduos. Em qualquer circunstância, uma decisão envolve a liberdade de escolha entre alternativas, caso contrário, seria uma imposição.

Na visão racional da economia, toda escolha implica na comparação entre alternativas, prevalecendo aquela que maximiza a função de utilidade do indivíduo ou da firma. Esta comparação pode ocorrer de diversas formas; ela pode ser feita entre alternativas concorrentes, ou com a mesma alternativa ao longo do tempo e, até mesmo, entre uma alternativa e um padrão (bechmarking). Em suma, o produto final da contabilidade é uma informação útil apenas no momento em que o usuário utilizá-la para fazer uma escolha que sempre envolve a comparação entre alternativas.

Com base neste raciocínio, a principal finalidade da informação contábil no processo decisório de seus usuários é servir de base para comparabilidade. A respeito disso, um dos principais representantes dos usuários da informação financeira, o CFA (2007), ressalta que os investidores não tomam decisões de investir ou não em determinada companhia no escuro. Pelo contrário, as decisões sobre investimento envolvem a ponderação das oportunidades e a escolha de um portfólio adequado ao seu perfil de risco e retorno. Portanto, fazer comparações é uma parte crítica do processo decisório sobre investimentos. A SEC (2000) compartilha da mesma opinião e afirma que quando um investidor julga o mérito e a comparabilidade dos investimentos, a alocação eficiente de capital é facilitada e a confiança do investidor é incentivada. Hendriksen (1967) também assevera que o principal objetivo da comparabilidade é auxiliar a tomada de decisões dos investidores e, assim, assistir o correto funcionamento do mercado, provendo um meio adequado pelo qual os acionistas consigam julgar os resultados das atividades em que investiram. Libby, Libby e Short (2009) vão ainda longe e afirmam que sem comparabilidade não existe nem análise financeira, pois não faria sentido analisar algo que não possa ser comparado.

No ambiente dos relatórios financeiros, não obstante exista uma discussão sobre o nível de importância da comparabilidade em relação a outras características qualitativas da informação contábil 
(Schuetze, 1994) e até mesmo a possibilidade de sua obtenção ser questionável (Durocher \& Gendron, 2011; Zhang \& Andrew, 2010), é inegável que ela cumpre um papel relevante no correto funcionamento de uma economia de mercado, pois isto foi comprovado empiricamente por diversos trabalhos a respeito desse tema (DeFranco, Kothari \&Verdi, 2011; Campbell \& Yeung, 2013; Barth, Landsman, Lang \& Williams, 2012; Peterson, Schmadebeck \& Wilks, 2012; DeFond, Hu, Hung \& Li, 2011; Fang, Maffett \& Zhang, 2013; Barth, Landsman, Lang \&Williams, 2013; Brochet, Jagolinzer \& Riedl, 2013; Kim, Kraft \& Ryan, 2013; Fang, Li, Xin \& Zhang, 2012; Sohn, 2011).

Embora a comparabilidade seja uma característica da informação fundamental para o correto funcionamento do mercado, ela ainda não é compreendida de modo claro no meio dos usuários nem dos preparadores dos relatórios financeiros (para exemplos ver trabalhos de Delloitte \& IBRI, 2011; FIPECAFI \& Ernst Young, 2013; e o artigo de Fernandes, 2013). O Brasil passou recentemente por um processo de alteração regulatória nas suas práticas de relatórios financeiros com a adoção do padrão internacional de contabilidade. A principal vantagem deste padrão em relação ao anterior é que ele permite ao gestor um maior nível de subjetividade nas suas decisões contábeis e isto faz com que os números contábeis passem a transparecer de modo mais adequado o racional econômico subjacente aos seus negócios. Este aspecto possui a tendência de melhorar a comparabilidade dos relatórios financeiros, mas não a sua uniformidade, pois os gestores possuem o incentivo a reconhecer as características peculiares das suas atividades, o que tende a diminuir a uniformidade dos demonstrativos financeiros (para comprovação do racional subjacente a tal afirmação ver Dye \& Verrecchia, 1995).

Não obstante as pesquisas sobre comparabilidade tenham demonstrado um ganho na medida tanto externo quanto interno com a adoção do padrão internacional (para comprovação empírica ver Ribeiro, 2014; Yip \& Young, 2012), na prática a visão sobre este ganho é cercada de ceticismo.

Em uma pesquisa realizada pela Delloitte junto com o Instituto Brasileiro de Relação com Investidores (IBRI) (2011) foram entrevistados profissionais da área de Relações com Investidores, ficou claro nos resultados que os mesmos não acreditam em uma melhora da comparabilidade após a adoção do padrão internacional no Brasil. Em outro trabalho realizado em parceria pela Fundação Instituto de Pesquisas Contábeis, Atuariais e Financeiras (FIPECAFI) e Ernst \& Young (E\&Y) (2013), um dos pontos levantados pelos pesquisadores foi o aumento da variabilidade das escolhas contábeis dos gestores após adoção do padrão International Financial Reporting Standards (IFRS) e como isto poderia ter prejudicado a comparabilidade dos balanços. Segundo as próprias entidades, "a forma de aplicação de algumas normas IFRS ainda não é um consenso entre os participantes do mercado, o que de certa forma levanta dúvidas sobre a comparabilidade das demonstrações contábeis".

Uma possível justificativa para esta visão mais pessimista sobre o ganho de comparabilidade dos demonstrativos contábeis pós-IFRS reside no fato da confusão conceitual que existe entre comparabilidade e uniformidade. Em um texto escrito no jornal Valor Econômico ficou bem clara a concepção deste vínculo, Fernandes (2013) afirma que "os IFRS suprem com excelente desempenho duas funções essenciais relacionadas às informações financeiras: transparência e uniformidade de divulgação, o que garante a comparabilidade".

Na academia também se observa uma confusão entre os conceitos até mesmo na formulação empírica de suas medidas. Alguns autores (Reina, Reina \& Silva, 2014; Ribeiro, Carmo \& Carvalho, 2013; Fabiano, 2012) chamam de comparabilidade medidas que capturam apenas a uniformidade dos relatórios financeiros. Tais medidas são embasadas, muitas vezes, em características similares nas práticas de divulgação ou nas escolhas contábeis entre diferentes companhias (os chamados T-index), mas não 
conseguem traduzir de modo adequado a comparabilidade dos relatórios financeiros segundo a sua estrutura conceitual, pois medem apenas a sua uniformidade.

Não obstante exista uma extensão em que os conceitos de comparabilidade e uniformidade sejam convergentes (May, 1938), na prática eles podem ser significativamente diferentes. O objetivo desta pesquisa é elucidar tais diferenças e testar empiricamente se, no ambiente dos relatórios financeiros, a comparabilidade e a uniformidade apresentam o mesmo comportamento durante o processo de alteração regulatória que ocorreu na contabilidade brasileira com a adoção do padrão internacional. Os resultados obtidos com este trabalho podem clarificar as diferenças entre os dois conceitos e ajudar tanto aos usuários das informações financeiras, quanto aos pesquisadores, a entender que comparabilidade e a uniformidade não devem ser confundidas na prática pois elas representam significados diferentes. Este artigo foi dividido em 5 partes, além desta introdução a segunda parte trouxe o referencial teórico com o conceito de comparabilidade e de uniformidade e as pesquisas anteriores. Na terceira e quarta partes foram apresentados o método e os resultados obtidos. Por fim, na quinta parte foram feitas algumas considerações finais.

\section{REFERENCIAL TEÓRICO}

\subsection{CONCEITO DE COMPARABILIDADE, UNIFORMIDADE E A SUA RELAÇÃO}

No ambiente dos relatórios financeiros, o Financial Accounting Standard Board (FASB) (2010) e IASB (2010) conceituam a comparabilidade como uma característica qualitativa a qual permite que os usuários identifiquem e compreendam similaridades dos itens e diferenças entre eles. Diferentemente de outras características qualitativas, a comparabilidade não está relacionada com um único item. $\mathrm{A}$ comparação requer no mínimo dois itens. (FASB, 2010; IASB, 2010)

Esta visão de comparabilidade foi inicialmente desenvolvida no comitê Trueblood que teve a incumbência de definir os objetivos dos relatórios financeiros (Miller, 1978; Merino \& Coe, 1978). Como destacado em tal estudo, comparabilidade significa ter similaridades reportadas do mesmo modo e desigualdades reportadas diferentemente ("to have like things reported alike, and unlike things reported differently").

Com base neste conceito, na dimensão dos relatórios financeiros, a comparabilidade é atingida quando existe um reflexo equivalente das circunstâncias econômicas entre duas companhias ou da mesma companhia ao longo do tempo, ou seja, as companhias devem possuir uma base de mensuração e uma apresentação equivalente de suas realidades econômicas.

Para os usuários das informações financeiras serem hábeis a efetivamente comparar o desempenho econômico e financeiro de uma companhia com outra ou da mesma companhia ao longo do tempo é necessário que as companhias sujeitas ao mesmo evento econômico e às mesmas condições institucionais reconheçam e mensurem os fatos patrimoniais de modo similar. Apenas a divulgação dos números do mesmo modo não garante a comparabilidade; é preciso, também, que eles representem o mesmo objetivo (Simmons, 1967).

Já a uniformidade, de acordo com Powell (1965), significa estar em conformidade com uma regra ou algo não variável. Na visão DeFond et al. (2011), isto se traduz em simplesmente requerer das firmas a adoção do mesmo conjunto de padrões contábeis de forma homogênea. 
Simmons (1967), por sua vez, aponta que a uniformidade é restritiva e presume uma ausência completa de variação na preparação e apresentação dos relatórios financeiros. Este autor também ressalta que estas restrições atingem a base de mensuração e avaliação do balanço assim como a suas técnicas de evidenciação. Simmons (1967) reforça que a comparabilidade entre demonstrativos contábeis pode ser vista como o objetivo final dos relatórios financeiros e a uniformidade é apenas parcialmente compatível com esta visão.

Deste modo, a uniformidade pode produzir comparabilidade em algumas situações e não produzir em outras. Procedimentos contábeis uniformes para diferentes realidades econômicas prejudicam a comparabilidade entre os relatórios financeiros.

Esta visão também é compartilhada por Hendriksen (1967) e May (1938). De acordo com Hendriksen (1967), a uniformidade dos relatórios financeiros é considerada frequentemente como um objetivo em si só, sendo que a finalidade principal é a comparabilidade, não uniformidade. O objetivo da uniformidade é aumentar a comparabilidade dos relatórios financeiros, isto é, a uniformidade é relevante se contribuir com a obtenção da comparabilidade.

May (1938), por sua vez, aponta que a uniformidade deve ser encarada como uma ajuda possível para tornar a contabilidade mais valorizada, particularmente para o leitor leigo aos demonstrativos financeiros. $\mathrm{O}$ autor ressalta que a uniformidade não é necessariamente útil no mundo ideal, se os usuários dos demonstrativos financeiros pudessem confiar nos relatórios como uma interpretação de todos os fatos relevantes de uma organização feita de acordo com o melhor julgamento de pessoas honestas e competentes e exercida com um perfeito entendimento do propósito para qual tais relatórios deveriam ser utilizados, a uniformidade dos procedimentos contábeis não seria relevante, mas como tal cenário é improvável de ser obtido, ela acaba possuindo valor.

Segundo a American Institute of Certified Public Accountants (AICPA) (1991), a necessidade de comparabilidade não deve ser confundida com a simples uniformização de procedimentos. Uniformidade significa simplesmente requerer das firmas seguirem as mesmas regras e produzirem os mesmos resultados (DeFond et al., 2011; May, 1938; Hendriksen, 1967). Comparabilidade, por sua vez, é uma característica da relação entre dois ou mais itens com informações (Simmons, 1967). Desta forma, enquanto comparabilidade é o resultado desejado de se adotar um conjunto uniforme de padrões contábeis, somente a uniformidade não resulta, necessariamente, em comparabilidade. Para que isso acontecer, é necessário que os padrões uniformes sejam adotados com fidedignidade.

Por exemplo, se uma companhia alterar a forma com que consome os benefícios dos seus equipamentos industriais, o seu método de depreciação deverá mudar. Deste modo, ela vai deixar de aplicar a sua política contábil de forma consistente, o que vai diminuir a uniformidade dos seus números contábeis ao longo do tempo.

Por outro lado, o novo método representará o modus-operandi atual do consumo dos benefícios dos seus equipamentos e foi a melhor estimativa econômica possível feita pela administração do negócio. Neste caso embora tenha havido uma perda de uniformidade, a comparabilidade não diminuiu, pois a realidade econômica do negócio mudou.

Se a administração tivesse mantido a mesma taxa de depreciação, a uniformidade não seria prejudicada, mas haveria uma perda de comparabilidade, pois os números contábeis não representariam fidedignamente a realidade econômica da companhia, incapacitando a sua característica mais importante para a comparabilidade que é representar o desempenho econômico da companhia para o mercado. 


\subsection{PESQUISAS RELACIONADAS}

É provável que o trabalho mais relevante desta nova geração de pesquisas sobre a comparabilidade seja o estudo de DeFranco et al. (2011). A grande novidade do artigo de tais autores foi a criação de uma nova medida para comparabilidade: a similaridade da função contábil. Tal medida é específica de cada companhia, baseada em output e quantitativa. Ela foi muito bem aceita pela academia, pois a maioria dos trabalhos da nova geração relacionados com a comparabilidade utilizou tal medida como constructo principal ou como medida alternativa para análise de sensibilidade (Yip \& Young, 2012; Wu \& Zhang, 2010; Lang et al., 2010; Barth et al., 2013; Fang et al., 2013; Cascino \& Gassen, 2012; Neel, 2013; Liao, Sellhorn \& Skaife, 2012; Brochet et al., 2013; Barth et al., 2012; DeFond et al., 2011;Kim et al., 2013; Sohn, 2011; Fang et al. 2012).

Além de criar uma nova medida mais robusta para medir o nível de comparabilidade dos relatórios financeiros, DeFranco et al. (2011) testaram, em uma amostra de companhias norte americanas, até que ponto a comparabilidade está relacionada com a probabilidade de um analista escolher duas empresas mais comparáveis como pares e qual a sua relação com a acurácia e a dispersão das suas previsões.

DeFranco et al. (2011) acharam que a probabilidade de um analista, o qual estivesse seguindo uma companhia em particular, seguir também outra companhia do mesmo setor aumentaria com uma maior comparabilidade entre tais companhias. Mais especificamente, os resultados do seu estudo mostraram que o aumento de um desvio padrão na medida de comparabilidade aumentava a probabilidade do analista selecionar a companhia como par de $1 \%$ a 3\%. Além disso, as companhias classificadas como mais comparáveis eram, também, acompanhadas por uma quantidade maior de analistas ( 0.5 a mais em média). Em relação à acurácia e dispersão das previsões, foi constatado que um crescimento de 1 desvio padrão na medida de comparabilidade aumenta a acurácia das previsões em aproximadamente $23 \%$ e diminui a dispersão das previsões em torno de $27 \%$.

Os resultados do trabalho de DeFranco et al. (2011) foram submetidos a uma análise de sensibilidade com outras medidas de comparabilidade como earnings co-movement, similaridade da função contábil com retorno defasado e com o fluxo de caixa como proxy para evento econômico e se mostraram significativos para todas as medidas com exceção da covariação dos lucros que apresentou um comportamento distinto.

DeFranco et al. (2011) concluíram, com base nos resultados apresentados, que a comparabilidade é positivamente relacionada com a quantidade de analistas, com a acurácia de suas previsões e negativamente relacionada com a dispersão das mesmas. Isso comprova, segundo os pesquisadores, que os analistas, realmente, beneficiam-se, por meio de um menor custo de aquisição e processamento de informação, com o aumento da comparabilidade. Quanto à covariação do lucro, por outro lado, os resultados foram apenas confirmados para a quantidade de analistas. Para os benefícios relacionados com as previsões dos analistas não foi observado um efeito significativo, o que remete a uma diferença entre as duas medidas.

Além de DeFranco et al. (2011), Lang et al. (2010) também utilizaram as duas medidas de comparabilidade e de uniformidade na sua pesquisa. O objetivo do trabalho de Lang et al. (2010) foi testar se as normas internacionais aumentaram a comparabilidade entre companhias de diferentes nacionalidades em uma amostra de países que adotaram o padrão internacional entre os anos 2005 e 2008.

Para medir a comparabilidade, os autores utilizaram uma medida adaptada do modelo DeFranco et al. (2011) para companhias de diferentes países e uma medida de covariação dos lucros (earnings 
co-movement) a qual captura qualquer coisa que crie similaridade nos lucros, independentemente dos eventos econômicos subjacentes serem ou não similares. Conforme os resultados obtidos, os países que adotaram o padrão internacional experimentaram um aumento na covariação dos lucros das suas companhias com os pares setoriais internacionais, mas apresentaram uma diminuição na sua medida de comparabilidade que captura os efeitos das similaridades econômicas.

Com base nestes resultados, os autores afirmam que este aumento na medida de covariação do lucro é nocivo ao correto funcionamento do mercado, pois ele está associado a um decréscimo na cobertura de analistas, a uma piora na exatidão das suas previsões e a um aumento no bid-ask spread.

De forma conjunta, os resultados obtidos pelo trabalho de Lang et al. (2010) sugerem que a adoção das IFRS acresceu a covariação dos lucros entre companhias de diferentes países, mas tal aumento não foi traduzido em ganho de comparabilidade cross-country genuína, nem em melhorias na habilidade dos analistas em aprender com suas comparações. Independentemente da maior ou menor robustez dos resultados de Lang et al. (2010), o seu trabalho efetivamente mostrou que a medida de covariação do lucro não apresenta as mesmas características da comparabilidade genuína que captura a essência subjacente ao racional econômico das companhias. Esta medida relaciona-se mais com a simples uniformização de procedimentos, tornando os lucros mais similares, porém menos comparáveis.

\section{DESENHO DA PESQUISA}

Metodologicamente esta pesquisa pode ser classificada como descritiva e, predominantemente, quantitativa. Os dados são secundários e foram coletados com o auxílio do programa Economática ${ }^{\circledR} \mathrm{e}$ dos websites da Comissão de Valores Mobiliários (CMV) e da Bolsa de Valores de São Paulo (BOVESPA). As variáveis dependentes são a comparabilidade e a uniformidade e o objetivo da pesquisa é investigar como tais medidas se comportaram ao longo do tempo (2004 a 2012) para identificar as similaridades e as diferenças entre os seus comportamentos. Para medir a comparabilidade foi utilizada como métrica o modelo de similaridade da função contábil desenvolvido por DeFranco et al. (2011) e para medir a uniformidade foi utilizado o modelo de co-movimentação do lucro de Lang et al. (2010). Para testar os comportamentos das variáveis dependentes sobre a janela temporal, optou-se pela abordagem estatística de dados em painel.

\subsection{COMPARABILIDADE}

A medida de comparabilidade utilizada nesta pesquisa foi a similaridade da função contábil de DeFranco et al. (2011). O primeiro passo para composição de tal métrica foi estimar a função contábil individual de cada companhia com base nos 12 últimos trimestres utilizando a seguinte equação:

$$
R O A_{i t}=\alpha_{i}+\beta_{i} \text { Retorno }_{i t}+\varepsilon_{i t}
$$

em que:

$\mathrm{ROA}_{\mathrm{it}}=$ Lucro líquido trimestral sobre o ativo total final da empresa i no período $t$ não consolidado

Retorno $_{i t}=$ Retorno médio trimestral da empresa i no período $t$, calculado com base no preço de fechamento das ações ordinárias (quando não líquidas, Preferenciais) ajustado para proventos e desdobramentos. 
Depois de estimar os parâmetros das funções individuais, foi preciso projetar o ROA esperado $[E(R O A)]$ de cada empresa com base nas regressões obtidas. Primeiramente, foi necessário estimar o ROA esperado específico da companhia no período de acordo com a seguinte função

$$
E(R O A)_{i i t}=\widehat{\alpha}_{i}+\widehat{\beta}_{i} \text { Retorno }_{i t}
$$

Posteriormente foi calculado o ROA esperado da mesma empresa com os estimadores das outras companhias do mesmo setor, segundo a seguinte equação:

$$
E(R O A)_{i j t}=\widehat{\alpha}_{j}+\widehat{\beta}_{j} \text { Retorno }_{i t}
$$

A ideia é manter o evento econômico constante, usando os estimadores de uma companhia no evento econômico da outra, assim a medida de comparabilidade é a média da distância entre estas duas funções para cada trimestre [E(ROAiit) - E(ROAijt)]. De acordo com DeFranco et al. (2011), quanto mais próxima forem as duas funções, maior é a comparabilidade entre as companhias. Para medir a comparabilidade individual entre os pares foi calculada a média da proximidade de cada função por período (trimestre) de acordo com a seguinte fórmula:

$$
\operatorname{Compb}_{i j t}=-\frac{1}{12} \times \sum_{t-11}^{t}\left|E\left(R O A_{i i t}\right)-E\left(R O A_{i j t}\right)\right|
$$

em que:

$\mathrm{Compb}_{\mathrm{ijt}}=$ Medida de comparabilidade individual relativa da empresa i baseada na empresa j.

$E\left(R_{\text {iit }}\right)=$ Retorno sobre o ativo esperado da empresa i com base nos estimadores da empresa i e o retorno da empresa i no período $t$.

$E\left(R_{i j}\right)=$ Retorno sobre o ativo esperado da empresa i com base nos estimadores da empresa j e o retorno da empresa i no período $t$.

A medida de comparabilidade obtida conforme a equação acima é uma medida relativa, isto é, ela mede as distâncias médias entre as funções de duas empresas isoladas. Para se obter uma medida de comparabilidade individual geral com as pares do setor é necessário calcular a média destas distâncias entre as companhias de referência, conforme a seguinte equação:

$$
C O M P M_{i t}=\frac{\operatorname{Compb}_{i j t}}{n}
$$

em que:

$\mathrm{COMPM}_{\mathrm{it}}=$ Medida de comparabilidade individual de cada companhia em relação às suas pares do setor

$\mathrm{Compb}_{\mathrm{ijt}}=$ Medida de comparabilidade relativa de cada par de empresas.

$\mathrm{n}=$ Número de companhias no setor (ou sendo comparadas)

Em relação ao modelo original de DeFranco et al. (2011), foram feitas três adaptações neste trabaIho: no período de estimação das funções individuais (16 para 12 trimestres), no deflator para tirar o efeito tamanho (ativo total) e na medida de lucro utilizada (devido ao período de transição, a medida de lucro operacional originalmente utilizada possui elementos diferentes antes e depois das normas internacionais). Tanto nesta pesquisa quanto em outros trabalhos que também utilizaram adaptações 
similares (Yip \& Young, 2012; Lang et al. 2010; Cascino \& Gassen, 2012; Brochet et al., 2013) não foi percebido um viés significativo em relação à medida original de DeFranco et al. (2011).

\subsection{UNIFORMIDADE}

A uniformidade dos relatórios financeiros foi traduzida nesta pesquisa em um processo de mimetização dos resultados de duas companhias. Se duas companhias seguirem as mesmas políticas contábeis independentemente dos seus respectivos eventos econômicos, a variação de seus resultados deve ocorrer de modo similar.

De acordo com Lang et al. (2010), a covariação dos lucros captura qualquer circunstância que aumente a similaridade dos lucros, independentemente de os eventos econômicos serem ou não similares. Como foi observado na discussão sobre as diferenças entre a comparabilidade e a uniformidade, esta foi a principal característica encontrada na uniformidade. Portanto, quanto maior for o poder explicativo da variação do lucro de uma companhia em função da variação do lucro de outra companhia maior é a uniformidade entre elas (Lang et al., 2010). Neste trabalho esta relação foi modelada da seguinte maneira:

$$
\operatorname{Lucro}_{i t}=\Phi_{0 i j}+\Phi_{1 i j} \text { Lucro }_{j t}+\varepsilon_{i j t}
$$

em que:

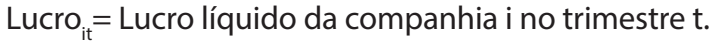

Lucro $_{\mathrm{jt}}=$ Lucro líquido da companhia j no trimestre t.

Neste modelo, a medida de comparabilidade é o poder explicado (R2) da série temporal do lucro de uma companhia i em função do lucro de uma companhia j. Quanto maior for o R2 desta regressão, maior será a uniformidade entre as companhias. Para atingir a uniformidade individual foi calculada a média dos $\mathrm{R} 2$ estimados com base nos pares dentro do setor, similarmente ao procedimento descrito na equação 5 . A quantidade de trimestres utilizados na série temporal foi a mesma da medida original (12).

\subsection{UNIVERSO PESQUISADO E COLETA DOS DADOS}

O universo de companhias utilizadas neste trabalho foi selecionado de forma intencional com o auxílio do programa Economática ${ }^{\oplus}$. O primeiro recorte necessário foi o país base da pesquisa. Tendo em vista que o objetivo do trabalho é identificar como a comparabilidade e a uniformidade se comportaram ao longo do tempo, optou-se pela utilização de apenas um país para limitar os efeitos das diferenças institucionais e econômicas sobre os resultados obtidos. O Brasil foi o país escolhido por ter acontecido nele um notório processo de flexibilização regulatória nos últimos anos, o que pode ressaltar as diferenças entre as duas medidas.

O segundo recorte foi feito nos setores de atividades econômicas analisados. Para a escolha dos setores foi utilizado a classificação do North American Classification System (NAICS) nível 2. O nível 2 do NAICS foi utilizado por grande parte dos trabalhos que investigaram a comparabilidade dentro dos setores (DeFranco et al., 2011; Yip \& Young, 2012; Lang et al., 2010). Os setores selecionados foram aqueles que apresentaram mais do que uma companhia com os dados trimestrais e anuais disponíveis do período de 2002 a 2012. Ao todo nove setores cumpriram tal condição, excluindo-se o setor bancário, pois os bancos não arquivam seus demonstrativos trimestrais de acordo com o padrão IFRS. 
No final foram analisados oito setores de atividades econômicas (construção pesada; empresas de eletricidade; indústria de equipamentos de transporte; indústria de produtos de metal; indústria de roupas; indústria química; siderurgia; telecomunicações). A Embraer e a Gerdau S.A. foram excluídas da pesquisa pois apresentam características destoantes das demais. A Embraer utiliza como moeda funcional o dólar e a Gerdau S.A. possui uma companhia subsidiária dentro do mesmo setor e estes dois aspectos poderiam distorcer a comparabilidade com as demais companhias nos seus respectivos setores. No fim foram analisadas um total de 54 empresas que possuíam dados na janela de tempo entre 2002 e 2012.

\subsection{ABORDAGEM ESTATÍSTICA E VARIÁVEIS}

Para cumprir o objetivo proposto por esta pesquisa, foi escolhida a abordagem estatística de dados em painel. O período de tempo analisado no trabalho foi 2004 a 2012, operacionalizado por meio de dummies de cada ano que representam o período de 2005 a 2012 com o ano de referência em 2004. O ano de 2004 foi escolhido como referência pois ele representa o período pré-adoção das IFRS. Em 2005 já foi criado o comitê de pronunciamentos contábeis (CPC) que pode ser considerado a primeira inciativa mais concreta no processo de internacionalizar o padrão contábil brasileiro que culminou com a adoção plena das IFRS em 2010.

Cabe ressaltar que para calcular a função contábil de cada companhia são necessários dados trimestrais de 2 anos anteriores ao período base de cálculo da medida. Portanto, para a medida de 2004, é necessário coletar dados de 2002 até 2004 (12 trimestres). Em decorrência disto, o recorte amostral envolveu empresas com dados dentro da janela de 10 anos (2002 a 2012), mas as diferenças foram apuradas apenas dentro do período 2004 a 2012.

No que tange às variáveis de controle, teoricamente, as medidas de comparabilidade e uniformidade empregadas nesta pesquisa possuem uma relação muito próxima com as teorias sobre escolhas contábeis e qualidade do lucro. Um resumo das variáveis e os trabalhos que embasaram as suas escolhas pode ser observado no Quadro 1.

Quadro 1: Variáveis utilizadas no modelo

\begin{tabular}{|c|c|c|}
\hline \multicolumn{3}{|l|}{ Variáveis Dependentes } \\
\hline Variável & Descrição & Operacionalização \\
\hline COMPR2 & Uniformidade individual média pares do setor. & Lucro $_{i t}=\Phi_{-o i j}+\Phi_{-} 1 \mathrm{ij}$ LuCro $_{\mathrm{jt}}+\varepsilon_{-\mathrm{ijt}}$ \\
\hline COMPM & Comparabilidade individual média pares do setor. & $\mathrm{ROA}_{\mathrm{it}}=\mathrm{a}_{\mathrm{i}}+\beta_{\mathrm{i}}$ Retorno $_{\mathrm{it}}+\varepsilon_{\mathrm{it}}$ \\
\hline \multicolumn{3}{|c|}{ Variáveis Independentes de Interesse e de controle } \\
\hline Variável & Descrição & Operacionalização \\
\hline ANO & $\begin{array}{l}\text { Período de flexibilização regulatória ocorrido no } \\
\text { Brasil. }\end{array}$ & $\begin{array}{l}\text { Dummies que representam os anos entre o período } 2005 \\
\text { a 2012. O ano de referência foi } 2004 \text {. }\end{array}$ \\
\hline LUCRO POR AÇÃO (LPA) & $\begin{array}{l}\text { Serve para representar a performance para os } \\
\text { acionistas. }\end{array}$ & (Lucro Líquido) / (Ações em Circulação) \\
\hline $\begin{array}{l}\text { GRAU ALAVANCAGEM OPERACIONAL } \\
\text { (GAOP) }\end{array}$ & $\begin{array}{l}\text { É uma medida de desempenho operacional e da } \\
\text { estrutura de custos da companhia. }\end{array}$ & (Lucro Bruto / \\
\hline \multicolumn{3}{|l|}{$\begin{array}{l}\text { (Lucro Bruto - Despesa com vendas e } \\
\text { Despesa administrativas) }\end{array}$} \\
\hline ENDIVIDAMENTO (END) & Serve para medir o risco de quebra de covenants. & (Dívida Bruta) / (Patrimônio Líquido) \\
\hline CRESCIMENTO (CRESC) & $\begin{array}{l}\text { Aumento ou diminuição percentual da receita bruta } \\
\text { da companhia em relação ao ano anterior. }\end{array}$ & (Receita Bruta_t - Receita Bruta_t-1) / Receita Bruta_t-1 \\
\hline PRICE BOOK RATIO (PBR) & $\begin{array}{l}\text { Mede a expectativa de crescimento do negócio para } \\
\text { o mercado. }\end{array}$ & (Valor de Mercado) / (PL contábil) \\
\hline TAMANHO (TAM) & $\begin{array}{l}\text { Ativo total da companhia transformado para sua base } \\
\text { logarítima. }\end{array}$ & Ln Ativo total \\
\hline RISCO & Beta anual da companhia (365 dias). & | Beta $\mid$ \\
\hline INTENSIDADE CAPITAL (INTCAP) & Mede o nível de tangibilidade do capital. & (Ativos Imobilizados) / (Patrimônio Líquido) \\
\hline
\end{tabular}




\begin{tabular}{|l|l|l|}
\hline PRESENCA (PRES) & Mede liquidez. & $\begin{array}{l}\text { (Quant.dias com ações negociadas) / (Quant.dias totais } \\
\text { de pregão) }\end{array}$ \\
\hline GOVERNANÇA (GOV) & $\begin{array}{l}\text { Mede se a companhia fez adesão a algum nível de } \\
\text { governança corporativa da BOVESPA. }\end{array}$ & $\begin{array}{l}\text { Variável Dummy com 2 categorias: possui (1) ou não } \\
\text { possui (0) nível de governança }\end{array}$ \\
\hline CONCENTRAÇÃO (CONC) & $\begin{array}{l}\text { Percentual de ações ordinárias possuídas pelo maior } \\
\text { acionista da companhia. }\end{array}$ & $\begin{array}{l}\text { \% Ações Ordinárias concentradas na mão do maior } \\
\text { acionista }\end{array}$ \\
\hline BIG4 & Firma que auditou o balanço no período determinado & Variável Dummy com 2 categorias: (1)BIG4 (0)Não BIG4 \\
\hline TROCA AUDITOR (TAUD) & $\begin{array}{l}\text { Variável dicotômica que mede se a companhia trocou } \\
\text { de auditor em relação ao período anterior. }\end{array}$ & $\begin{array}{l}\text { Variável Dummy com 2 categorias: trocou (1) enão trocou } \\
\text { (0) a firma de auditoria }\end{array}$ \\
\hline SETOR & Classificação NAICS nível 2. & $\begin{array}{l}\text { Variável Dummy que representa os setores de atividade } \\
\text { econômica: (0) Energia Elétrica, (1) Construção Pesada, (2) } \\
\text { Ind. Eq. Transportes, (3) Ind. Eq. Metal, (4) Ind. Têxtil, (5) } \\
\text { Ind. Química, (6) Ind. Siderúrgica e (7) Telecomunicações }\end{array}$ \\
\hline
\end{tabular}

Nota. As variáveis de controle foram selecionadas de acordo com os seguintes trabalhos: Ribeiro (2014), DeFranco et al. (2011), Dechow, Ge \& Schrand (2010), Watts \& Zimmerman (1986), Ashbaugh, Collins, Kinney \& LaFond (2008), LaFond \& Roychowdhury (2008) e Lawrence, Minutti-Meza \& Zhang (2011).

Quanto ao modelo de painel, ele pode ser classificado como curto (maior quantidade de indivíduos do que de anos) e fortemente balanceado (todos os indivíduos foram mantidos ao longo dos anos). A técnica escolhida na estimação do modelo foi Ordinary Last Square (OLS), pois ela foi superior ao efeito aleatório segundo o teste LM de Breusch-Pagan.

O teste dos efeitos fixos não foi computado, pois o modelo trabalha com dados que não variam ao longo do tempo (ex. setor e governança). Isto iria derrubar tais controles e fugiria do objetivo da pesquisa. Além disto, o efeito temporal está controlado nas dummies que representam os anos analisados no trabalho.

Antes de processar os dados, foi feito um tratamento para detecção e tratamento de outliers multivariados, seguindo a técnica proposta por Hadi (1992). Para confirmar o efeito da aplicação do tratamento para as observações discrepantes foram rodados os modelos também sem a sua exclusão. Os resultados não apresentados demonstraram que não existe um viés significativo nas inferências da pesquisa com a presença dos outliers. Mesmo assim, como a ideia é comparar períodos optou-se pela exclusão dos dados discrepantes.

\section{APRESENTAÇÃO E ANÁLISE DOS RESULTADOS}

O primeiro ponto analisado por esta pesquisa foi o comportamento e as medidas de posição das variáveis utilizadas. Os resultados obtidos para medida de comparabilidade com base nas companhias do mesmo setor (COMPM =-2,634) (ver tabela 1) foram muito parecidos com aqueles obtidos por pesquisas feitas anteriormente em outros países. DeFranco et al. (2011), Fang et al. (2012) e Sohn (2011) encontraram médias de -2,7,-2,03 e -1,907 respectivamente em suas análises com companhias dos Estados Unidos. Já Peterson et al. (2012), que pesquisaram as companhias europeias, encontraram uma média de-2,597. Estes resultados comprovam que a medida de comparabilidade obtida neste trabalho está em consonância com as mesmas medidas alcançadas em âmbito internacional.

Tabela 1: Estatística descritiva das variáveis utilizadas na pesquisa

\begin{tabular}{|l|l|l|l|l|l|l|l|l|}
\hline VARIÁVEL & N & Média & Mediana & $\begin{array}{l}\text { Desvio Padrão } \\
\text { Overall }\end{array}$ & $\begin{array}{l}\text { Desvio Padrão } \\
\text { Between }\end{array}$ & $\begin{array}{l}\text { Desvio Padrão } \\
\text { Within }\end{array}$ & Min \\
\hline COMPM & 474 & $-2,63$ & $-2,28$ & 1,41 & 0,97 & 1,05 & $-7,89$ & $-0,33$ \\
\hline COMPR2 & 486 & 0,14 & 0,12 & 0,09 & 0,04 & 0,08 & 0,00 & 0,51 \\
\hline LPA & 473 & 1,34 & 0,79 & 2,29 & 1,84 & 1,42 & $-7,18$ & 10,10 \\
\hline GAOP & 452 & 1,07 & 1,2 & 1,24 & 1,09 & 0,73 & $-3,70$ & 4,83 \\
\hline ENDIV & 432 & 41,92 & 27,69 & 44,64 & 39,53 & 23,47 & 0,00 & 208,06 \\
\hline TAM & 486 & 21,38 & 21,5 & 1,65 & 1,63 & 0,36 & 17,58 & 25,44 \\
\hline
\end{tabular}




\begin{tabular}{|l|l|l|l|l|l|l|l|l|}
\hline CRESC & 451 & 0,10 & 0,1 & 0,18 & 0,07 & 0,17 & $-0,51$ & 0,69 \\
\hline PRES & 486 & 74,99 & 90,69 & 30,07 & 25,83 & 15,75 & 4,42 & 100,00 \\
\hline PBR & 460 & 1,57 & 1,31 & 1,32 & 1,13 & 0,82 & $-2,76$ & 6,20 \\
\hline RISCO & 477 & 0,95 & 0,94 & 0,40 & 0,38 & 0,13 & 0,09 & 2,83 \\
\hline INTCAP & 444 & 56,37 & 33,02 & 64,88 & 53,04 & 37,48 & 0,00 & 295,34 \\
\hline CONC & 475 & 62,43 & 59,44 & 25,01 & 23,37 & 9,12 & 6,77 & 99,97 \\
\hline ANO & 486 & & & & & & 2004 & 2012 \\
\hline SETOR & 486 & & & & & & 0 & 1 \\
\hline TAUDI & 486 & & & & & & 0 & 1 \\
\hline BIG4 & 486 & & & & & & 0 & 1 \\
\hline GOV & 486 & & & & & & 0 & 1 \\
\hline
\end{tabular}

Em relação à uniformidade, Lang et al. (2010) encontraram um resultado de 0,358 para o R2 médio das companhias que não adotaram as IFRS e um resultado de 0,364 para as companhias que adotaram. Comparativamente às companhias brasileiras analisadas, a medida de uniformidade em âmbito internacional apresentou uma média muito maior (quase 3 vezes). Este aspecto pode estar relacionado com a transição regulatória da contabilidade brasileira para o padrão internacional. No trabalho de Lang et al. (2010) ele não fez um estudo longitudinal.

Ao analisar o comportamento dos desvios padrões das variáveis, percebe-se que, embora as variáveis dependentes (COMPR2 e COMPM) tenham uma variação maior ao longo do tempo (within) do que entre indivíduos, quase todas as demais variáveis independentes apresentam uma variação between maior. Este aspecto pode ser um indício da utilização de um modelo de painel com efeitos aleatórios ou POLS. Como o teste LM confirmou a adequação do POLS frente ao efeito aleatório, esta foi a técnica utilizada para estimar o modelo. Para confirmar a escolha entre os modelos, foram estimados modelos com efeitos fixos e aleatórios em comparação ao POLS. Os resultados não apresentados confirmam uma semelhança entre os estimadores e entre a significância das variáveis em ambos os modelos.

Figura 1: Comportamento da Comparabilidade média e da Uniformidade média durante o período analisado na pesquisa

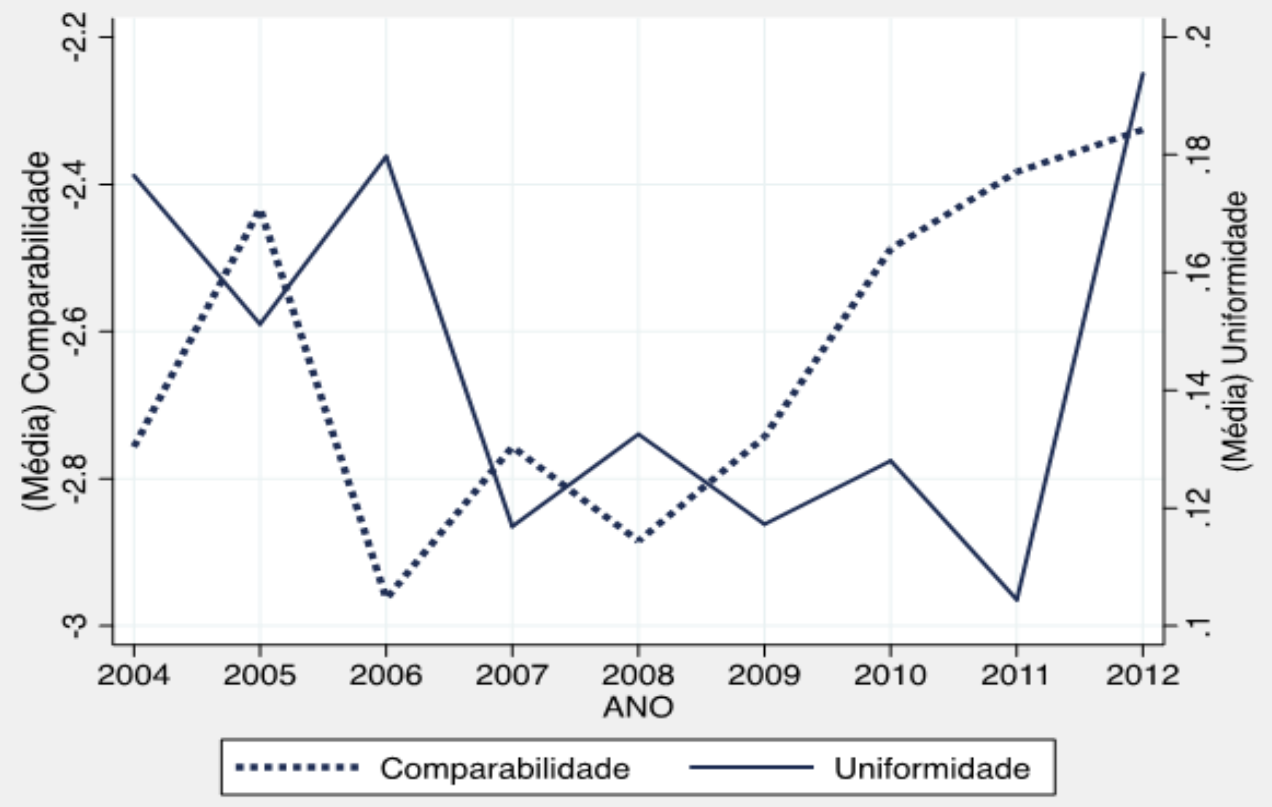


Quanto ao comportamento das medidas de comparabilidade e uniformidade durante o período de transição regulatória, graficamente foi identificada uma variação distinta entre elas. Enquanto a medida de comparabilidade apresentou uma queda após 2005 e uma tendência de alta após 2008, a uniformidade apresentou uma queda acentuada em 2006 e só se recuperou após 2011 depois do segundo ano de adoção das normas internacionais.

Percebe-se com base na Figura 1 que, não obstante a uniformidade tenha acompanhado o comportamento da comparabilidade (embora com o lag) durante o período de transição, no período de maior utilização de accruals discricionários [entre 2009 e 2011 segundo a tese de Silva (2013)] houve um descasamento significativo entre as medidas. Enquanto a comparabilidade obteve um ganho com o aumento da discricionariedade, a uniformidade obteve uma perda que só foi revertida após 2011, dois anos depois da adoção do padrão internacional no Brasil. Esta reversão no comportamento da uniformidade pode ser um indício da ocorrência de um efeito de mimetização na preparação dos demonstrativos financeiros. Durante o período de transição regulatória é normal existirem mais interpretações divergentes sobre como os eventos econômicos se incorporam nos números contábeis, ainda mais para um regime de normas baseadas em princípios, onde as companhias perderam um pouco a referência regulatória do como fazer.

Neste ambiente é natural que uma companhia busque a outra de maior sucesso no mercado como forma de benchmarking para equiparar as suas políticas contábeis, o que cria um movimento de mimetização independente da realidade econômica a qual a companhia está sujeita e isto consequentemente aumenta a uniformidade, mas não necessariamente a comparabilidade, pois a mesma depende do evento econômico.

Para confirmar as diferenças observadas graficamente entre os comportamentos da comparabilidade e da uniformidade foi rodado um teste de correlação entre as duas variáveis. O resultado obtido foi de 0,0211 ( $p$-value igual a 0,646). Lang et al. 2011 também encontraram uma correlação muito fraca e não significativa entre as duas medidas $(0,025)$.

Tabela 2: Estatística descritiva das variáveis utilizadas na pesquisa

\begin{tabular}{|c|c|c|c|c|c|c|}
\hline \multirow{2}{*}{ Anos } & \multicolumn{3}{|c|}{ Comparabilidade (COMPM) } & \multicolumn{3}{|c|}{ Uniformidade (COMPR2) } \\
\hline & 1 & ॥ & III & I & ॥ & III \\
\hline 2005 & $0,3238^{* *}$ & $0,4079^{*}$ & & $-0,0252$ & $-0,0278$ & $-0,0315^{* *}$ \\
\hline 2006 & $-0,2063$ & 0,0467 & & 0,0032 & 0,0051 & \\
\hline 2007 & 0,0001 & 0,4247 & & $-0,0596^{* * *}$ & $-0,0777^{* * *}$ & $-0,0851^{* * *}$ \\
\hline 2008 & $-0,1279$ & 0,0068 & & $-0,0439 * *$ & $-0,0611^{* *}$ & $-0,0616^{* * *}$ \\
\hline 2009 & 0,0135 & 0,4249 & & $-0,0592^{* * *}$ & $-0,0677^{* * *}$ & $-0,0726^{* * *}$ \\
\hline 2010 & 0,2690 & 0,4458 & & $-0,0484^{* * *}$ & $-0,0586^{* *}$ & $-0,0594^{* * *}$ \\
\hline 2011 & 0,3736 & $0,6233^{*}$ & $0,4122^{* *}$ & $-0,0720^{* * *}$ & $-0,0783^{* * *}$ & $-0,0844^{* * *}$ \\
\hline 2012 & 0,4310 & $0,7516^{* *}$ & $0,5496^{* * *}$ & 0,0173 & 0,0144 & \\
\hline \multicolumn{7}{|c|}{ Controles } \\
\hline LPA & & 0,0165 & & & $-0,0031^{*}$ & $-0,0048^{* *}$ \\
\hline GAOP & & $0,2547^{* * *}$ & $0,2719^{* * *}$ & & $-0,0034$ & \\
\hline END & & 0,0035 & & & $-0,0001$ & \\
\hline TAM & & 0,0364 & & & $0,0150^{* * *}$ & $0,0100^{* * *}$ \\
\hline CRESC & & 0,0000 & & & 0,0127 & \\
\hline PRES & & 0,0036 & & & 0,0000 & \\
\hline PBR & & $-0,2166^{* * *}$ & $-0,1935^{* * *}$ & & $-0,0015$ & \\
\hline RISCO & & $-0,0143$ & & & $-0,0002$ & \\
\hline INTCAP & & $-0,0012$ & & & 0,0002 & \\
\hline
\end{tabular}




\begin{tabular}{|c|c|c|c|c|c|c|}
\hline CONC & & $-0,0044$ & & & 0,0001 & \\
\hline TAUDI & & 0,0560 & & & $-0,0221^{* *}$ & \\
\hline GOV & & $-0,0596$ & & & $-0,0107$ & \\
\hline BIG4 & & $-0,1052$ & & & $-0,0031$ & \\
\hline CONSTR & & $1,4687^{* * *}$ & $1,6426^{* * *}$ & & 0,0351 & \\
\hline TRANSP & & $-0,6828^{* *}$ & $-0,4627^{* *}$ & & $0,0339^{*}$ & \\
\hline METAIS & & 0,4928 & & & $0,0380^{*}$ & \\
\hline TÊXTIL & & $-1,6001^{* * *}$ & $-1,4203^{* * *}$ & & 0,0054 & \\
\hline QUÍMICA & & $-1,3407^{* * *}$ & $-1,2491^{* * *}$ & & $0,0413^{* *}$ & \\
\hline SIDERU & & $-0,4166$ & & & $0,0639^{* * *}$ & $0,0488^{* * *}$ \\
\hline TELECOM & & $-0,6694$ & & & $-0,0433^{* * *}$ & $-0,0542^{* * *}$ \\
\hline CONS & $-2,7565^{* * *}$ & $-3,3355$ & $-2,4759^{* * *}$ & $0,1765^{* * *}$ & $-0,1445$ & $-0,0177$ \\
\hline R2 & 0,0244 & 0,3606 & 0,2881 & 0,1156 & 0,2902 & 0,2509 \\
\hline Root MSE & 1,4002 & 1,0945 & 1,12 & 0,0845 & 0,0777 & 0,0777 \\
\hline $\mathrm{F}$ & $3,57^{* * *}$ & $114,06^{* * *}$ & $87,19^{* * *}$ & $6,02 * * *$ & $21,24^{* * *}$ & $27,82^{* * *}$ \\
\hline VIF & 1,81 & 2,02 & - & 1,78 & 1,99 & - \\
\hline Ramsey & 2,49 & 1,09 & 0,69 & $19,60^{* * *}$ & $10,08^{* * *}$ & $13,17^{* * *}$ \\
\hline$N$ & 474 & 350 & 350 & 486 & 353 & 353 \\
\hline
\end{tabular}

Nota. ${ }^{*}$ ** / ** denotam significância bi-caudal nos níveis 0,10 / 0,05 / 0,01 respectivamente. As regressões acima foram estimadas com OLS e foi utilizada a opção com erros padróes robustos clusterizados nos individuos. No modelo III stepwise foi utilizado o critério backward com um cutoff de 0.05 . A constante absorveu as seguintes categorias para as variáveis dummy: $A N O=2004, T A U D I=N a ̃ o, G O V=N a ̃ o, B I G 4=$ Não, SETOR = Energia. Os modelos I foram obtidos a partir das seguintes equaçôes: $C O M P M_{i t}=\beta_{0}+\beta_{1} 2005_{i t}+\beta_{2} 2006_{i t}+\beta_{3} 2007_{i t}+\beta_{4} 2008_{i t}+\beta_{5} 2009_{i t}+\beta_{6} 2010_{i t}+\beta_{7} 2011_{i t}+\beta_{8} 2012_{i t}+\varepsilon_{i t t}$ e COMPR2 $=\beta_{0}+\beta_{2} 2005_{i t}+\beta_{2}$

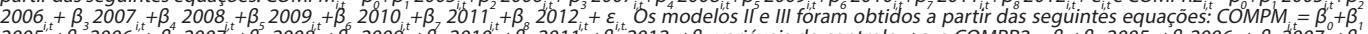

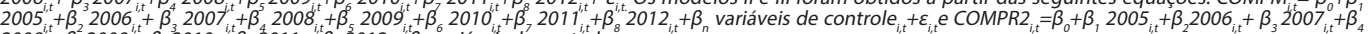
$2008_{i, t}, \beta_{5}^{2} 2009_{i, t}+\beta_{6} 2010_{i, t}, \beta_{7} 2011_{i, t}+\beta_{8} 2012_{i, t}+\beta_{n}$ variáveis de controle $e_{i, t}+\varepsilon_{i, t}$

Na Tabela 2 estão expostos os resultados do comportamento da comparabilidade individual média (COMPM) e da uniformidade individual média (COMPR2) dos relatórios financeiros ao longo do tempo. $\mathrm{O}$ modelo com todas as variáveis de controle (II) para comparabilidade e para uniformidade apresentaram R2 de 0,36 e 0,29 respectivamente. Este número é similar aos obtidos por trabalhos relacionados com esta pesquisa (DeFranco et al., 2011 e Lang et al., 2011 encontraram um coeficiente aproximado de 0,2 nos seus modelos de comparabilidade).

Percebe-se na análise da Tabela 2, que o efeito temporal nas variáveis dependentes foi totalmente distinto entre os anos 2005 e 2012. Enquanto a comparabilidade não apresentou uma diferença significativa em relação ao ano de 2004 durante o período de transição e teve um aumento significativo após a adoção das IFRS para os anos 2011 e 2012 (no modelo III), a uniformidade apresentou perdas significativas em relação a todo o período de pré-adoção e pós-adoção do padrão internacional (exceto pelos anos 2006 e 2012 que não apresentaram diferenças significativas em relação a 2004).

Quanto aos controles, os comportamentos foram os esperados pela teoria exposta no Quadro 1 e nota-se que existe um efeito negativo para troca de auditor em relação à uniformidade (o que já era esperado pois, muitas vezes, os vieses contábeis variam entre as firmas de auditoria e isto pode influenciar o preparador). Adicionalmente, ficou evidente uma diferença entre os setores para as medidas de comparabilidade e uniformidade [para aprofundar tais efeitos e para eventuais justificativas ver a pesquisa de Ribeiro (2014)].

Ao traçar um paralelo entre os resultados obtidos com esta pesquisa e os achados dos trabalhos que utilizaram o T-index no Brasil como em Reina et al. (2014) e Fabiano (2012), pode-se inferir que a medida utilizada em tais pesquisas possui um comportamento muito mais próximo da uniformidade do que da comparabilidade. Fabiano (2012) mostrou graficamente no seu trabalho que o comportamento do T-index para as empresas que utilizaram o padrão IFRS durante o período 2006 e 2010 foi negativo corroborando a medida de uniformidade desta pesquisa. O pesquisador encontrou também 
um efeito significativo do tamanho das companhias sobre a uniformidade das escolhas contábeis, resultado similar ao deste trabalho.

Em âmbito internacional, os trabalhos de DeFranco et al. (2011) e de Lang et al. (2011), assim como nesta pesquisa, também comprovaram a diferença entre a similaridade da função contábil (medida de comparabilidade) e a covariação dos lucros (medida de uniformidade). Sobre isto, Lang et al. (2011) afirmam que a comparabilidade e a uniformidade (do modo como foram medidas nesta pesquisa) são realmente construtos distintos e que ambos podem apresentar diferentes implicações para os usuários dos demonstrativos contábeis. Os autores ressaltam que, enquanto a comparabilidade "verdadeira" aumenta a utilidade da informação para os seus usuários por meio da maior cobertura de analistas e da maior acuracidade e menor dispersão nas suas previsões, a simples uniformidade refletida na covariação dos lucros é prejudicial, pois ilude os usuários a acreditarem que as informações são iguais enquanto a realidade econômica na verdade é distinta. DeFranco et al. (2011) vai na mesma linha e aponta que a covariação dos lucros (uniformidade) pode atrair analistas a escolher companhias similares como pares de comparação, mas ela não está associada a uma melhoria nas suas habilidades de previsões e a comparabilidade, por outro lado, está.

Os resultados obtidos com esta pesquisa vão na mesma linha destes trabalhos e apontam para as companhias analisadas que o comportamento da comparabilidade é muito diferente do comportamento da uniformidade dentro do período analisado. Estes achados corroboram a teoria que destacam as diferenças entre os dois conceitos (Miller, 1978; May, 1938; Hendriksen, 1967; Simmons, 1967; Cole et al., 2012; DeFond et al., 2011) e fornecem evidências empíricas de que a comparabilidade e a uniformidade apresentam tanto diferenças teóricas quanto práticas.

\section{CONSIDERAÇÕES FINAIS}

O objetivo desta pesquisa foi verificar se existem diferenças significativas entre o comportamento da comparabilidade e da uniformidade dos relatórios financeiros durante um período de transição regulatória da contabilidade brasileira. Os resultados encontrados apontam que o aumento da flexibilização regulatória ocorrida no cenário contábil nacional teve um efeito negativo sobre a uniformidade, mas positivo sobre a comparabilidade.

Estes resultados demonstram que a comparabilidade não pode ser confundida a qualquer forma de uniformização de procedimentos. Enquanto na uniformidade presume-se apenas a divulgação da informação do mesmo modo ou com o mesmo efeito patrimonial sem levar em consideração as diferenças entre os modelos econômicos das companhias, a comparabilidade "genuína" (Zeff, 2007) é obtida quando empresas sujeitas ao mesmo evento (modelo) econômico reconhecem, mensuram e evidenciam tal evento do mesmo modo (Schipper, 2003; Simmons, 1967), ou seja, o output do modelo contábil de determinada companhia é o mesmo de empresas sujeitas aos mesmos fenômenos econômicos. FASB (2010) e IASB (2010) também concordam com esta visão do conceito de comparabilidade, pois, segundo tais entidades, a comparabilidade é uma característica qualitativa que permite aos usuários identificar e compreender tanto similaridades quanto diferenças entre itens.

Portanto, a comparabilidade "genuína" depende de duas variáveis: do evento econômico e da forma como uma empresa traduz este evento para dentro da sua contabilidade. São mais comparáveis as companhias que, dado o mesmo evento econômico, apresentem resultados contábeis similares. 
Apenas a similaridade da evidenciação ou das políticas contábeis adotadas não garantem a comparabilidade. Para ela acontecer, é necessário que os gestores os quais enfrentam as mesmas características econômicas possuam os mesmos incentivos institucionais e particulares para traduzir tais eventos em números contábeis.

Os resultados apresentados nesta pesquisa corroboram este ponto de vista e são um indicativo aos usuários e preparadores dos relatórios financeiros que apenas divulgar a mesma informação não necessariamente se traduz em uma maior comparabilidade dos relatórios financeiros, para isto acontecer o evento econômico subjacente ao que vai ser reportado deve ser o mesmo. Portanto, as companhias que copiam as políticas contábeis de outras companhias ou da norma contábil estabelecida não possuem um ganho comparabilidade garantido apenas pela evidenciação ou pela uniformidade. Para que o ganho ocorra é necessário que elas expressem de modo adequado as entrelinhas econômicas dos seus negócios mesmo que isto signifique em divulgar uma política contábil diferente das demais companhias. Como foi visto, a comparabilidade não depende apenas das similaridades, mas também das diferenças entre as atividades econômicas.

Este aspecto ressalta a importância da compreensão do negócio antes de se analisar um relatório financeiro. Em um ambiente de relatórios financeiros mais uniformes o viés das escolhas contábeis dos gestores estão presentes nos regulamentos, pois eles devem ser mais específicos quanto ao que as companhias podem ou não fazer em termos contábeis, o que pode afetar a tradução dos eventos econômicos em números contábeis. Já em um ambiente de relatórios mais comparáveis o viés das escolhas recaem sobre os gestores para que os mesmos consigam transmitir com maior fidedignidade os seus modelos de negócios. O investidor e o analista devem ajustar os seus modelos de análise com base nestas características e tentar identificar previamente se a uniformidade dos relatórios prejudica ou não a sua comparabilidade. Adicionalmente, os resultados obtidos podem, do mesmo modo, auxiliar os reguladores na hora de decidir qual nível de especificidade aplicar aos regulamentos. Uma norma muito específica tende a aumentar a uniformidade dos relatórios e uma mais flexível a sua comparabilidade, que é a característica desejável.

Para futuras pesquisas encoraja-se trabalhos relacionados com a comparabilidade dos relatórios financeiros utilizando-se uma métrica que consiga capturar o sentido mais adequado do conceito como a similaridade da função contábil de DeFranco et al. (2011).

\section{REFERÊNCIAS}

American Institute of Certified Public Accountants - AICPA. (1991). Professional Standards. AICPA: New York.

Ashbaugh, H., Collins, D. W., Kinney, W. R., \& Lafond, R. (2008). The effect of sox internal control deficiencies and their remediation on accrual quality. The Accounting Review, 83(1), 217-250.

Barth, M. E., Landsman, W. R., Lang, M. H., \& Williams, C. D. (2013). Effects on comparability and capital markets benefits of voluntary adoption of IFRS by US firms: Insights from voluntary adoption of IFRS by non-US firms. Working Paper. 
Barth, M. E., Landsman, W. R., Lang, M., \& Williams, C. (2012). Are IFRS-based and US GAAP-based accounting amounts comparable? Journal of Accounting and Economics, 54(1), 68-93.

Brochet, F., Jagolinzer, A. D., \& Riedl, E. J. (2013). Mandatory IFRS Adoption and Financial Statement Comparability. Contemporary Acc Res, 30(4), 1373-1400.

Campbell, J. L., \& Yeung, E. P. (2013). Limited attention to accounting comparability? An analysis of price contagion around earnings restatements. Working Paper.

Cascino, S., \& Gassen, J. (2012). What drives the comparability effect of mandatory IFRS adoption? Working Paper.

Chartered Financial Analysts - CFA. (2007). A comprehensive business reporting model: Financial reporting for investors. London: CFA Institute Centre for Financial Market Integrity.

Dechow, P., Ge, W., \& Schrand, C. (2010). Understanding earnings quality: A review of the proxies, their determinants and their consequences. Journal of Accounting and Economics, 50(2-3), 344-401.

DeFond, M., Hu, X., Hung, M., \& Li, S. (2011). The impact of mandatory IFRS adoption on foreign mutual fund ownership: The role of comparability. Journal of Accounting and Economics, 51(3), 240-258.

DeFranco, G., Kothari, S. P., \& Verdi, R. S. (2011). The Benefits of Financial Statement Comparability. Journal of Accounting Research, 49(4), 895-931.

Delloitte e Instituto Brasileiro de Relações com Investidores (IBRI). (2011). IFRS em prática: Os RI'S na Grande Virada da Contabilidade Brasileira. Reino Unido: Delloitte Touche Tohmatsu.

Durocher, S., \& Gendron, Y. (2011). IFRS: On the docility of sophisticated users in preserving the ideal of comparability. European Accounting Review, 2, 233-262.

Dye, R. A., \& Verrecchia, R. (1995). Discretion vs. uniformity: Choices among GAAP. The Accounting Review, 70(3), 389-415.

Fabiano, D. (2012). O nível de comparabilidade contábil dos países do BRIC. Programa de Pós-Graduação em Administração, Faculdade de Gestão e Negócios, Universidade Federal de Uberlândia.

Fang, V. W., Maffett, M., \& Zhang, B. (2013). Foreign institutional ownership and the global convergence of financial reporting practices. Working Paper.

Fang, X., Li, Y., Xin, B., \& Zhang, W. J. (2012). Accounting comparability and loan contracting. Working Paper. 
Fernandes, Edison (2013, Outubro 22). Contabilidade Como Ferramenta de Política Econômica. Valor Econômico. Caderno Fio da Meada. Recuperado em 18 Outubro, 2016, de http://www.valor.com.br/ legislacao/fio-da-meada/3311418/contabilidade-como-ferramenta-de-politica-economica.

Financial Accounting Standards Board - FASB. (2010). Statement of financial accounting concepts No. 8: Conceptual framework for financial reporting. Norwalk, CT, September.

FIPECAFI, \& EY. (2013). Análise sobre o IFRS no Brasil (3a ed.).

Hail, L., C. Leuz, \& P. Wysocki. (2010). 'Global accounting convergence and the potential adoption of IFRS by the U.S. (Part I): Conceptual underpinnings and economic analysis.' Accounting Horizons, 24, 355-394.

Hadi, A. S. (1992). Identifying multiple outliers in multivariate data. Journal of the Royal Statistical Society, 54(3), 761-771.

Hendriksen, E. S. (1967). Toward greater comparability through uniformity of accounting principles. The New York Certified Public Account, 37(2), 105-115.

International Accounting Standard Board - IASB. (2010). The conceptual framework for financial reporting. IASB. London.

Joos, P. \& Leung, E. (2011). Investor perceptions of the potential IFRS adoption in the United States. Working paper, Tilburg University.

Kim, S., Kraft, P., \& Ryan, S. G. (2013). Financial statement comparability and credit risk. Working Paper. Stern School of Business.

Lafond, R., \& Roychowdhury, S. (2008). Managerial ownership and accounting conservatism. Journal of Accounting Research, 46(1), 101-135.

Lang, M. H., Maffett, M., \& Owens, E. (2010). Earnings Comovement and Accounting Comparability: The Effects of Mandatory IFRS Adoption. Working Paper.

Lawrence, A., Minutti-Meza, M., \& Zhang, P. (2011). Can big 4 versus non-big 4 differences in audit-quality proxies be attributed to client characteristics? The Accounting Review, 86(1), 259-286.

Liao, Q., Sellhorn, T., \& Skaife, H. A. (2012). The cross-country comparability of IFRS earnings and book values: evidence from France and Germany. Journal of International Accounting Research, 11(1), 155-184.

Libby, R.; Libby, P.A. \& Short, D.G. (2009). Financial Accounting. 6º ed. New York: McGraw-Hill//rwin.

May, G. (1938). Uniformity in accounting. Harvard Business Review, XVII(1), 1-8. 
Miller, P. B. W. (1978). A new view of comparability. The Journal of Accountancy, August, 70-77.

Merino, B. D., \& Coe, T. L. (1978). Uniformity in accounting: A historical perspective. The Journal of Accountancy, August, 62-69.

Neel, M. (2013). Accounting comparability and economic outcomes of mandatory IFRS Adoption. Working Paper.

Peterson, K., Schmardebeck, R., \& Wilks, J. T. (2012). Accounting comparability and earnings attributes. Working Paper.

Powell, W. (1965). Putting uniformity in financial accounting into perspective. Law \& Contemporary Problems, 27(934), 674-689.

Reina, D.R.M, Reina, D. Silva, S.F. (2014). Comparabilidade da Informação Contábil em Empresas Brasileiras após a Adoção das Normas Internacionais de Contabilidade. Revista Contemporânea de Contabilidade. 11(23), 77-94.

Ribeiro, A.M. (2014). Poder discricionário do gestor e comparabilidade dos relatórios financeiros: Uma análise dos efeitos da convergência do Brasil às IFRS. Departamento de Contabilidade e Atuária da Universidade de São Paulo.

Ribeiro, A. M., Carmo, C. H. S., \& Carvalho, L. N. G. De. (2013). Evidenciação ambiental: regulamentar é a solução para falta de comparabilidade e objetividade? Revista de Contabilidade e Organizações, 17, 6-21.

Schipper, K.(2003). Principles-Based accounting standards. Accounting Horizons, 17(1),61-72.

Schuetze, W. P. (1994). What is the future of mutual recognition of financial statements and is comparability really necessary? The European Accounting Review, 3(2), 330-334.

Scott, W. R. (2009). Financial accounting theory (5a ed.). Toronto: Pearson.

Securities and Exchange Commission - SEC. (2000). SEC concept release: International Accounting Standards. Washington, D.C: SEC.

Securities and Exchange Commission - SEC. (2010). Commission Statement in Support of Convergence and Global Accounting Standards.

Silva, R. L. M. (2013). Adoção completa das IFRS no Brasil: Qualidade das demonstrações contábeis e o custo de capital próprio. Departamento de Contabilidade e Atuária da Universidade de São Paulo. 
Simmons, J. (1967). A Concept of Comparability in Financial Reporting. The accounting review, October, 680-692.

Sohn, B. C. (2011). The effect of accounting comparability on earnings management. Working Paper. City University of Hong kong.

Tweedie, S. D. (2007). Can global standards be principle based? The Journal of Applied Research in Accounting and Finance, 2(1), 3-8.

Watts, R. L., \& Zimmerman, J. L. (1986). Positive accounting theory. New Jersey: Prentice Hall Career \& Technology.

Wu, J. S., \& Zhang, I. (2010). Accounting integration and comparability: Evidence from relative performance evaluation around IFRS adoption. Working Paper. The Bradley Policy Research Center Financial Research and Policy.

Yip, R. W. Y., \& Young, D. (2012). Does mandatory IFRS adoption improve information comparability? The Accounting Review, 87(5), 1767-1789.

Zeff, S. a. (2007). Some obstacles to global financial reporting comparability and convergence at a high level of quality. The British Acc Review, 39(4), 290-302.

Zhang, Y., \& Andrew, J. (2010). Land in China: Re-considering Comparability in Financial Reporting. Australasian Acc Business Finance Journal, 4(1), 53-76. 